\title{
ON ICE-CRYSTAL PRODUCTION BY AEROSOL PARTICLES
}

\author{
By N.H. Fletcher \\ C.S.I.R.O., Radiophysics Laboratory, Sydney, Australia
}

(Manuscript received 10 June 1958)

\begin{abstract}
A general theory of the action of small aerosol particles as ice-crystal nuclei is developed and its predictions compared with experiment. It is found possible to decide under what conditions a given particle will act as a sublimation or as a freezing nucleus, and it is concluded that silver iodide generally behaves as a sublimation nucleus. The size effect is used to explain the activity distribution of silver iodide smoke, and this in turn explains the time lag observed in the nucleation of ice crystals by this smoke.
\end{abstract}

\section{Introduction}

A considerable amount of experimental data on the action of aerosol particles as ice-crystal nuclei is now available in the literature of cloud physics. Though many of the results appear to be in conflict, there is a substantial amount of information which can be accepted as reliable.

It is the aim of this paper to put forward several general theoretical results which, when applied to cases in which the results of experiment are fairly unambiguous, give a good explanation of the observed phenomena. In many cases, the discussion will also be found to suggest reasons for the disagreement between observations which are apparently in conflict.

It is hoped that the points raised will help to clarify some of the important considerations involved in the design of laboratory experiments and in the discussion of results in this field.

\section{Nucleation thermodynamics and the size effect}

The theory of the nucleation of new phases is now well established, and a comprehensive review has been given by Dunning (1955). In the absence of foreign nuclei, the rate of homogeneous formation, per unit volume, of embryos of critical size of the new phase is given by

$$
J=B \exp \left(-\Delta G^{*} / k T\right),
$$

where $B$ is a kinetic constant and $\Delta G^{*}$ is the free energy required to form a critical embryo. $\Delta G^{*}$ is just the height of the free-energy barrier separating groups of molecules of the parent phase from large stable embryos of the new phase which can grow into droplets or crystals. Equation (1) is then a simple classical rate equation for the surmounting of this barrier.

To evaluate $\Delta G^{*}$, we consider the free energy required to form a sphere of the new phase of radius $r$. Let $\Delta G_{v}$ be the free-energy difference, per unit volume of the new phase, between new and old phases of infinite extent, and let $\sigma$ be the surface free energy of the interfacé. Then

$$
\Delta G=\frac{4}{3} \pi r^{3} \Delta G_{v}+4 \pi r^{2} \sigma .
$$

To find the maximum value $\Delta G^{*}$ of this free energy, we set the differential of $\Delta G$ with respect to $r$ equal to zero. This gives us the radius $r^{*}$ of the critical embryo

$$
r^{*}=-2 \sigma / \Delta G_{v}
$$

and yields the value

$$
\Delta G^{*}=16 \pi \sigma^{3} / 3\left(\Delta G_{v}\right)^{2} .
$$

When a foreign substance is present, the new phase, can form on the foreign catalytic surface rather than forming homogeneously within the old phase. An important parameter in this process is the angle of contact $\theta$ between the new phase 2 and the catalyst 3 immersed in the old phase 1 . In terms of surface free energies, $\theta$ is given by

$$
m=\cos \theta=\left(\sigma_{13}-\sigma_{23}\right) / \sigma_{12} .
$$

The quantity $m$ as defined here is the function of $\theta$ which appears in the nucleation equations.

Analogously with (1) we have the rate equation for heterogeneous nucleation on a plane surface, per unit area,

$$
J=B^{\prime} \exp \left(-\Delta G^{*} / k T\right),
$$

where the constant $B^{\prime}$ differs from $B$ of (1). $\Delta G^{*}$ is found exactly as before, assuming the embryo to form a spherical cap with contact angle $\theta$ on the foreign surface. The surface and volume terms involve $m$ and the final result is

$$
\Delta G^{*}=16 \pi \sigma^{3} f(m) / 3\left(\Delta G_{v}\right)^{2},
$$

where

$$
f(m)=(2+m)(1-m)^{2} / 4 .
$$


The function $f(m)$ varies from surface to surface and it is this which determines the relative nucleation efficiency of various substances.

We now require an explicit expression for the bulk free energy difference $\Delta G_{v}$. This can be derived from general thermodynamic considerations which we will not go into here. If the new phase 2 is a liquid and the parent phase 1 a supersaturated vapor, then it can be shown (Glasstone, 1947) that

$$
\Delta G_{v}=-\frac{k T}{V_{2}} \log \frac{p_{1}}{p_{\infty}}
$$

where $V_{2}$ is the volume occupied by a molecule of the liquid, $p_{\infty}$ is the saturated vapor pressure over a plane surface of the liquid and $p_{1}$ is the actual vapor pressure of the phase 1 .

If, on the other hand, phase 2 is a solid, then as discussed by Turnbull and Vonnegut (1952) the solid embryo will in general be in a state of elastic strain because its lattice constant differs from that of the nucleating substrate. This elastic strain $\epsilon$ contributes a term to $\Delta G_{v}$ so that

$$
\Delta G_{v}=-\frac{k T}{V_{2}} \log \frac{p_{1}}{p_{\infty}}+c \epsilon^{2} .
$$

There may also be a contribution to the surface free energy $\sigma_{23}$ due to dislocations between the two lattices at their interface. This effect is, however, automatically taken into account in the definition of $m$.

We now introduce a new complication which is, in fact, very important to the present discussion. The nucleating substances considered above had plane surfaces and so were of infinite extent. In practice, nucleating particles are small and often of approximately spherical shape. If we make the approximation of assuming spherical nucleating particles of radius $R$, then instead of (6) and (7) we have, as the author has shown elsewhere (Fletcher, 1958a), for the rate of formation of embryos per nucleating particle

$$
\begin{aligned}
J & =B^{\prime} 4 \pi R^{2} \exp \left(-\Delta G^{*} / k T\right) \\
\Delta G^{*} & =16 \pi \sigma^{3} f(m, x) / 3\left(\Delta G_{v}\right)^{2}
\end{aligned}
$$

where $x$ is the ratio of the radius $R$ of the particle to the radius $r^{*}=-2 \sigma / \Delta G_{v}$ of the critical embryo, i.e.,

$$
x=R / r^{*}=-R \Delta G_{v} / 2 \sigma .
$$

The function $f(m, x)$ is given by

$$
\begin{aligned}
f(m, x)= & +\left(\frac{1-m x}{g}\right)^{3} \\
& +x^{3}\left[2-3\left(\frac{x-m}{g}\right)+\left(\frac{x-m}{g}\right)^{3}\right] \\
& \quad+3 m x^{2}\left[\frac{x-m}{g}-1\right],
\end{aligned}
$$

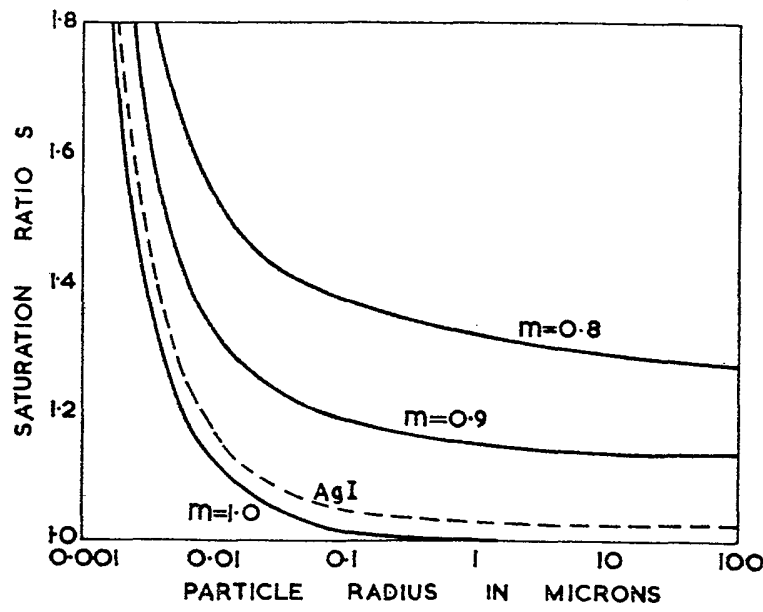

FIG. 1. Saturation ratio $S=p / p_{\infty}$ necessary to produce droplets on spherical nuclei of radius $R$ at $0 \mathrm{C}$. The parameter $m$ is the cosine of the angle of contact of water on the nucleus material. Approximate curve for silver iodide is shown.

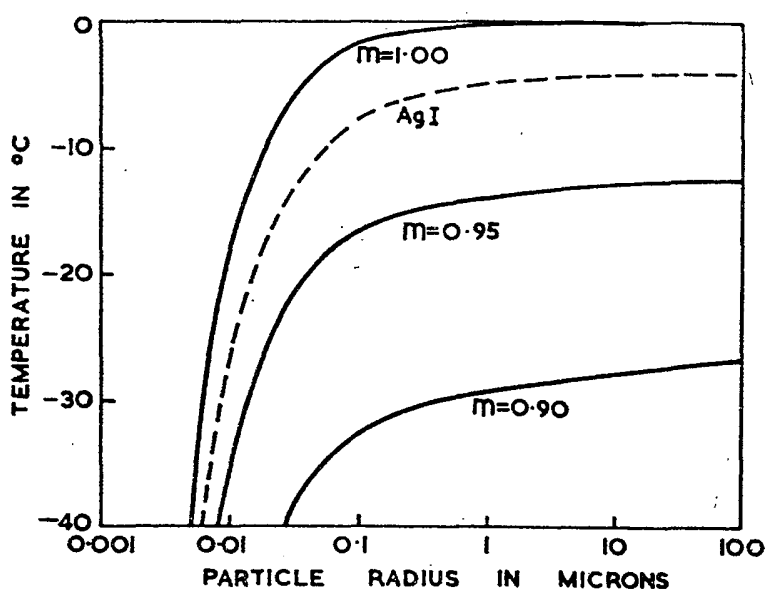

FIG. 2. Super-cooling temperature $T$ in $\operatorname{deg} \mathrm{C}$ required to produce ice crystals by sublimation onto spherical nuclei of radius $R$ at water saturation. The parameter $m$ is the cosine of the angle of contact of ice on the nucleus material assuming no elastic strain. Approximate curve for silver iodide is shown.

where

$$
g=\left(1+x^{2}-2 m x\right)^{\frac{1}{2}} .
$$

Of course $f(m, x)$ reduces to $f(m)$ when $x \rightarrow \infty$.

Equations (11) through (15), together with the relevant expression for $\Delta G_{v}$, allow us to calculate the complete behavior of the nucleation process. Apart from the nucleating parameters $m$ and $\epsilon$, the only other unknown is the kinetic constant $B^{\prime}$. Unfortunately, this is not well known as its calculation depends on the details of the nucleating process. The expression (11) for the nucleation rate is, however, dominated by the exponential factor, so that large uncertainties in $B^{\prime}$ have very little effect upon the critical value of $\Delta G_{v}$.

Calculation of specific cases involves considerable labor but is simple in principle. For most of the 


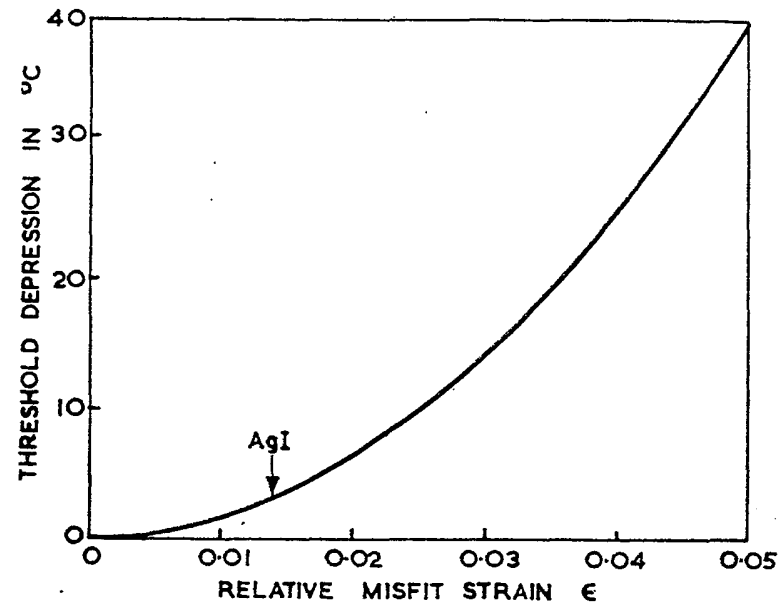

FIG. 3. Additional super-cooling $\Delta T$ required if nucleation is coherent upon a substrate with relative misfit $\epsilon$

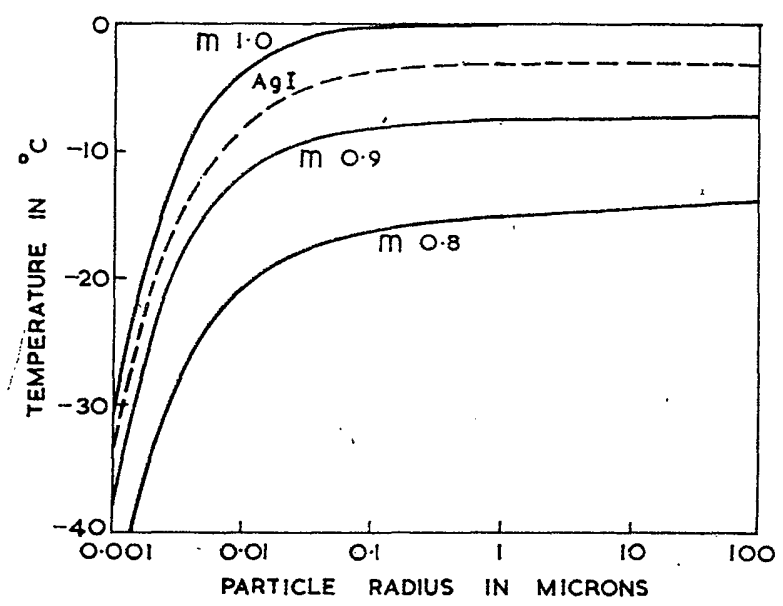

FIG. 4. Super-cooling temperature $T$ in deg $C$ required to produce ice crystals by freezing onto spherical nuclei of radius $R$. The parameter $m$ is the cosine of the angle of contact of ice on the nucleus material under water, assuming elastic strain to be zero. An approximate curve for silver iodide is also shown.

processes in which we are interested, $B^{\prime}$ is about $10^{25}$ $\mathrm{cm}^{-2} \mathrm{sec}^{-1}$, and we may take an appreciable rate of nucleation to be $J=1$ embryo per particle per second, the exact choice of this quantity being unimportant. For a given particle radius $R$, equation (11) now gives a specific value for $\left(\Delta G^{*} / k T\right)$ and this can be related to supersaturation or supercooling with the aid of equations (12) through (15). The results will be essentially unchanged for any other reasonable definition of an appreciable nucleation rate.

In fig. 1, we have shown the supersaturations necessary to produce water droplets on spherical nuclei at $0 \mathrm{C}$. The curves are not very temperature sensitive over the small temperature ranges usually encountered (say $-20 \mathrm{C}$ to $+20 \mathrm{C}$ ). It should be remembered that there are usually so many hygroscopic or wettable nuclei in the atmosphere that even in strong updraughts the supersaturation is most unlikely to exceed 1 per cent (Howell, 1949). This value then sets a limit on the types of nuclei which can ordinarily become active in the atmosphere.

Fig. 2 shows a similar set of curves for the sublimation of ice onto nucleating particles from an environment at water saturation. A value of $100 \mathrm{erg} \mathrm{cm}^{-2}$ was assumed for $\sigma_{12}$. This is only an approximate value but is sufficiently accurate so that the curves should not be in error by more than about 10 per cent. In this calculation, it has been assumed that the elastic strain $\epsilon$ is zero. A correction for elastic strain energy can be made if the strain and the elastic constants of ice are known. For lattices like AgI which approximate quite closely the ice lattice, it is probably correct to assume coherent nucleation, in which case the strain $\epsilon$ is equal to the misfit between the two lattices. This case has been considered for plane substrates by Turnbull and Vonnegut (1952). To a good approximation, the presence of strain merely depresses all the curves of fig. 2 by the same amount in temperature. Fig. 3, which is drawn from the data of Turnbull and Vonnegut, neglecting the elastic anisotropy of ice, gives an approximate value for this depression as a function of lattice misfit.

Fig. 4 shows a similar set of curves for freezing upon a particle suspended in water. Again, if elastic strain is present, the correction indicated in fig. 3 should be applied. A value of $20 \mathrm{erg} \mathrm{cm}^{-2}$ was assumed for $\sigma_{12}$ in this case. This is only a very rough approximation and may be seriously incorrect. The curves of fig. 4 are therefore not very reliable and may be in error by as much as a factor of 2. They still serve, however, as a semi-quantitative guide.

We shall comment on all these curves in more detail later when we come to compare their predictions with experiment.

\section{Condensation, sublimation, and freezing}

There has been, and continues to be, considerable argument as to whether various aerosol particles produce ice crystals by a process of sublimation or of freezing. Though freezing may not involve a macroscopic droplet, it does involve the condensation of sufficient water on the nucleus so that a nice embryo can be formed entirely within it and, for this reason, it is clearly a two stage process whilst sublimation involves only a single process. We shall neglect the possibility that water covers the nucleus because of a collision with a water droplet. This may in fact happen in some cases but does not appear likely to be the dominant mechanism.

The question of sublimation or freezing can therefore be resolved by comparing the rates of sublimation and condensation. Once condensation has taken place, sublimation is impossible and freezing will follow if the temperature is sufficiently low. 
Fig. 5 summarizes the situation for a typical nucleating agent with a small contact angle for both ice and water. We shall neglect for the moment the surface drawn in broken lines. The nucleation surfaces for condensation and sublimation are those on which the nucleation process takes one second. Below and to the right of these surfaces, the nucleation process is very rapid; above and to the left, it is very slow. Elastic strain has been neglected so that melting occurs at $0 \mathrm{C}$ as shown. For other materials, the two surfaces will be very similar but displaced along the $S$-axis to allow for different contact angles.

This figure is only useful in a general way and, to obtain specific information, it is better to calculate cross sections using the formulae of section 2. For example, fig. 1 gives an $R-S$ cross section of the condensation surfaces for various materials at $T=0 \mathrm{C}$ whilst fig. 2 gives an $R-T$ cross section of the sublimation surfaces at $S=1$. We can, however, on examination of fig. 5, make several general deductions about the behavior of nucleating particles, among which are the following:

1. Almost all sufficiently large particles (say. greater than 1 micron) can act as sublimation nuclei at slightly less than water saturation $(S=1)$ provided the temperature be lowered sufficiently. Decreasing $S$ by 1 per cent, however, requires that $T$ be lowered by about $1 \mathrm{C}$ if the activity is to be constant.

2 . In the size range from about 0.01 to 0.1 micron, even particles wet by water require considerable supersaturation to nucleate droplets but may act as sublimation nuclei at moderate supercoolings and $S$ values near 1.0. In this important size range, therefore, insoluble particles can only be active as sublimation nuclei in the atmosphere.

3. At very small supercoolings and slight supersaturation with respect to water, condensation is more rapid than sublimation. The particles may therefore act as condensation nuclei and, if their properties are suitable, as freezing nuclei.

Whilst this discussion, together with more detailed calculations, takes account of most situations, there remains one case which is not adequately covered. This is the situation in which a nucleating particle is suddenly subjected to a large supersaturation at a temperature below $0 \mathrm{C}$. From fig. 5, both condensation and sublimation proceed at fast rates, but it may be important in some cases to decide which process is dominant. Since the kinetic coefficient $B^{\prime}$ of (11) is almost the same for condensation and sublimation, the relative rates may be simply determined by comparison of $\Delta G^{*}$ for the two processes under the same conditions.

Without going into this calculation in detail, it leads to a further surface dividing regions of preferred sublimation from regions of preferred condensation.

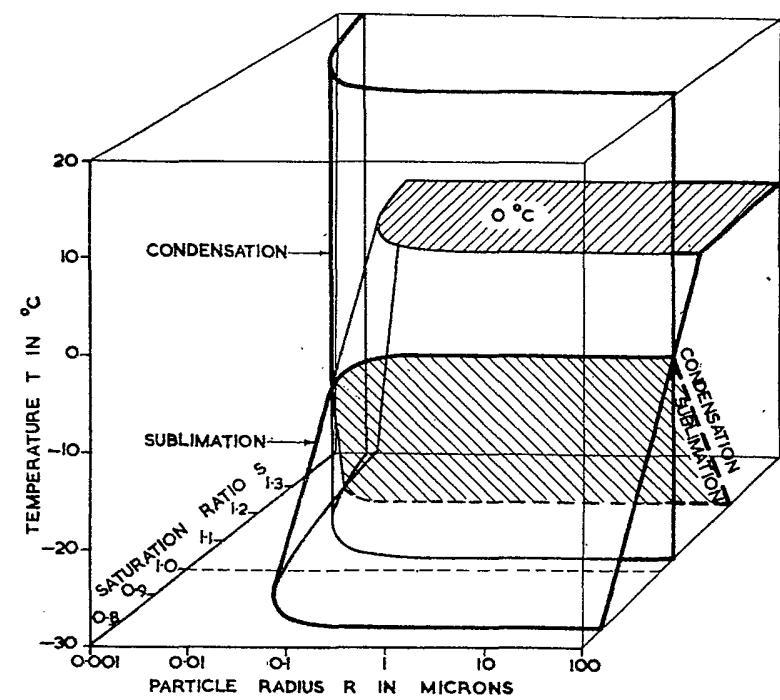

FIG. 5. Relations between particle radius $R$, saturation ratio with respect to water $S$, and temperature $T$ required to nucleate water droplets by condensation or ice crystals by sublimation on a nucleating material with contact angle about $5 \mathrm{deg}$ for water and about 25 deg for ice.

This surface is indicated with broken lines in fig. 5 and passes, of course, through the intersection of the other two surfaces. As indicated in the figure, condensation is preferred above the surface-i.e., towards high supersaturations and warm temperatures, and sublimation below the surface, for small supersaturations and low temperatures.

The details of fig. 5 depend to some extent upon our choice of $100 \mathrm{erg}$ per $\mathrm{cm}^{2}$ for the surface free energy of ice, but none of our conclusions are altered except by small numerical factors provided only that this surface free energy is greater than that of water. We have avoided Krastanow's (1940) assumption of polyhedral ice embryos as being perhaps unreal (Fletcher, 1958a) and, in any case, of less importance than the difference in surface free energy between ice and water.

\section{Comparison with experiment}

In principle, the surface free energies determining the contact angle and hence $m$ can be deduced from fundamental molecular data; but in practice this has not yet been possible. Liquid contact angles can be measured on macroscopic crystal faces and should give a reasonable approximation to the correct values, but this has not yet been done with solids. An empirical approach is available, however, which allows us to estimate these cases, as we shall see later.

Condensation.-Our discussion has been implicitly restricted to solid insoluble nuclei, and we shall not consider nucleation by droplets and hygroscopic solids despite their prime importance in atmospheric condensation. This omission is justified since experiments show such nuclei to be relatively inefficient in ice formation. 


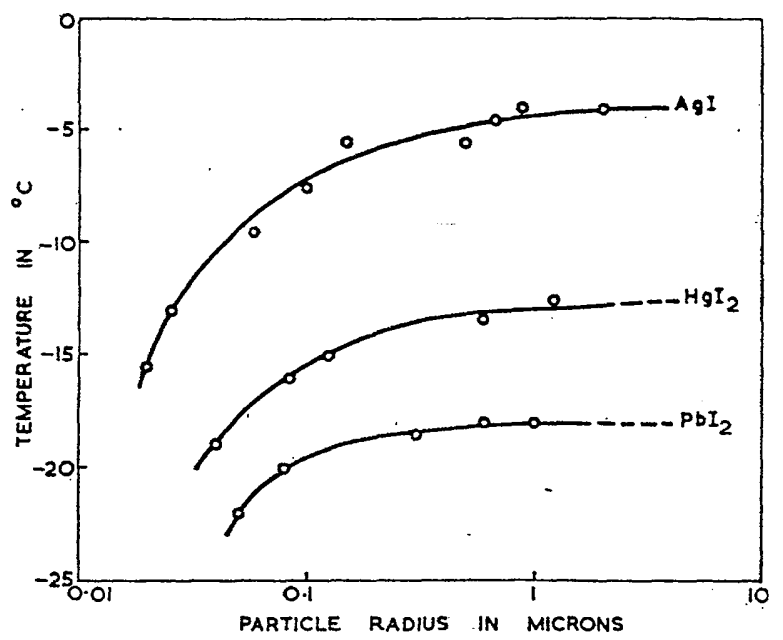

FIG. 6. Nucleation temperature as a function of size for smokes of various materials (redrawn from Sano, et al, 1956).

Discussions of condensation have usually made the assumption that all solid nuclei are wet by water-i.e., that $m=1$. We shall content ourselves with an examination of the important case of silver iodide; here, this assumption is invalid.

Large crystals and powders of AgI behave in a typically hydrophobic manner, floating easily upon water. A rough estimate of contact angle made by putting a droplet of water on a face of a large crystal indicated a contact angle of about $20 \mathrm{deg}$, whilst an estimate by the air bubble method indicated a smaller but definitely non-zero contact angle ${ }^{1}$. This accords with the hydrophobic behavior of the substance and its very slight solubility.

An aerosol of $\mathrm{AgI}$ was produced by heating a small quaritity of the pure salt in a clean pyrex tube through which was passing carefully filtered air. Some of this aerosol was allowed to settle on a glass slide for subsequent microscopic examination which revealed a density of about $5 \times 10^{5} \mathrm{~cm}^{-3}$ of particles between 0.5 - and $2-\mu$ diam and an additional density of smaller particles which was hard to estimate.

After evaluating the background of nuclei produced by the heated pyrex tube, the silver-iodide aerosol was introduced into a small expansion-type cloud chamber at room temperature and its behavior examined ${ }^{2}$ as the supersaturation was increased. Little or no fog was produced at supersaturations up to 2 per cent, but between 2 and 3 per cent the fog density increased sharply to an estimated $10^{6}$ droplets per $\mathrm{cm}^{3}$. The threshold was estimated to be at about 2.5 per cent supersaturation. This value should change negligibly down to temperatures of $-20 \mathrm{C}$.

\footnotetext{
1 Since this writing, Head and Sutherland of the Division of Physical Chemistry, C.S.I.R.O., have made a more accurate determination of contact angle between water and crystalline and polished silver-iodide surfaces. The equilibrium angle was found to be $10^{\circ} \pm 2^{\circ}$.

${ }_{2}$ The author is greatly indebted to Dr. S. Twomey of this laboratory for advice and assistance with this experiment.
}

Comparing this result with fig. 1 , it is seen that 1- $\mu$ particles show negligible size effect and one can deduce that $m \approx 0.98$ for these particles, implying a contact angle of $11 \mathrm{deg}$. This is in quite good agreement with the visual estimates made of the macroscopic contact angle.

The size effect has been investigated by many workers in connection with natural aerosols at high supersaturations, and reasonably good agreement with the simple theory (which corresponds to our case $m=1$ ) has been found. We can thus place considerable confidence in the curves calculated in fig. 1 for more general cases.

This result has very important consequences. Because of the presence of droplets and hygroscopic nuclei in the air, the supersaturation is unlikely to exceed 1 per cent and in clouds the figure may be considerably lower than this. This means that AgI particles injected into clouds do not act as condensation nuclei. This is true even for large particles, and from fig. 1 it is true a fortiori for the particles of $0.1-\mu$ diam and less usually used in cloud-seeding experiments. Thus, as is indicated in fact by the surfaces in fig. 5, silver iodide in the atmosphere must act as a sublimation rather than a freezing nucleus. We shall return to this point later.

Sublimation.-Though an estimate of the elastic strain $\epsilon$ involved in sublimation can be made from fig. 3 , information about $m$ values must be derived indirectly. To do this, we observe that nucleation by very large particles is size independent and the highest observed nucleation temperature thus fixes the right hand end of the appropriate curve in fig. 2 and using this with a correction from fig. 3 we find $m$. For example, AgI has a misfit of about 1.4 per cent which should lower the curve of appropriate $m$ by about 3C. The highest observed nucleation temperature for $\mathrm{AgI}$ smoke is about $-4 \mathrm{C}$ so that the uncorrected curve must reach about $-1 \mathrm{C}$ implying $m \approx 0.996$-i.e., an angle of contact of about $5 \mathrm{deg}$. The appropriate corrected curve is marked on fig. 2.

We should remark, in passing, that all large particles need not necessarily show the maximum activity. This is because our theory assumed perfectly spherical particles, whereas large particles are likely to have many surface irregularities which cause them to act as assemblages of small particles which are much less efficient.

Sano, Fujitani, and Maena (1956) have investigated the size effect for smokes of several substances with the results shown in fig. 6 . If we make the reasonable assumption that these substances all act as sublimation nuclei, then it is seen that these curves accord well with those for different values of $m$ and $\epsilon$ in our fig. 2 . The substances shown all melt at temperatures of a few hundred degrees. Results on smokes of refractory 
oxides show rather larger size effects which we may attribute to large deviations from simple spherical particles.

The size effect can also explain in detail the observed spread in nucleation temperature of silver iodide and other smokes. It is well known that the number of ice crystals produced by a given smoke increases greatly as the temperature of the supercooled cloud is lowered. This can be explained as the activation of successively smaller and smaller particles as the temperature is lowered.

The nucleation temperature distribution of various silver-iodide burners has been studied by Vonnegut (1949), Smith and Heffernan (1954), and Soulage (1955), among others. Their results differ in detail because of the different burners used, but agree that above $-10 \mathrm{C}$ the activity increases approximately exponentially as the temperature falls $(1 \mathrm{C}$ to $2 \mathrm{C}$ for a factor of 10) and that the activity saturates before about $-20 \mathrm{C}$. This agreement suggests that, despite the presence of sodium iodide and unburnt carbon in some of the smokes, they still behave essentially as pure evaporated silver iodide.

Size distributions determined for silver-iodide smoke by severál workers (Birstein, 1955 ; Sano and Fukuta, 1956; Sano et al, 1956) show that the number distribution is approximately normal when plotted against the logarithm of particle radius. This log-normal distribution is common for particles produced by many processes. The mean radius and standard deviation depend, of course, upon details of the smokeproduction technique.

Photomicrographs given by Smith and Heffernan (1954). for the output of their kerosene burner show a mean particle radius of about 0.03 micron though this is rather hard to estimate. Assuming a standard deviation similar to that of Sano and Fukuta (1956), we can then evaluate the activity distribution to be expected on the basis of the size effect. The result of this calculation is shown in fig: 7 where it is compared with the experimental curve. Ordinates have been adjusted by simply sliding the theoretical curve along the number axis for best fit. This is a valid procedure since a large percentage of the burner mass output is concentrated in relatively few large particles, about whose number there is scant information, It can be seen that agreement as to shape and saturation temperature is remarkably good. It is hoped to discuss the characteristics of the smokes of various types of burners in detail in a forthcoming publication.

Despite this good agreement, it is felt that contamination of the smoke by other components of the burning solution cannot fail to have considerable effect upon the details of its behavior.

An activity distribution of this type is to be expected for all smokes having sufficiently non-uniform particle

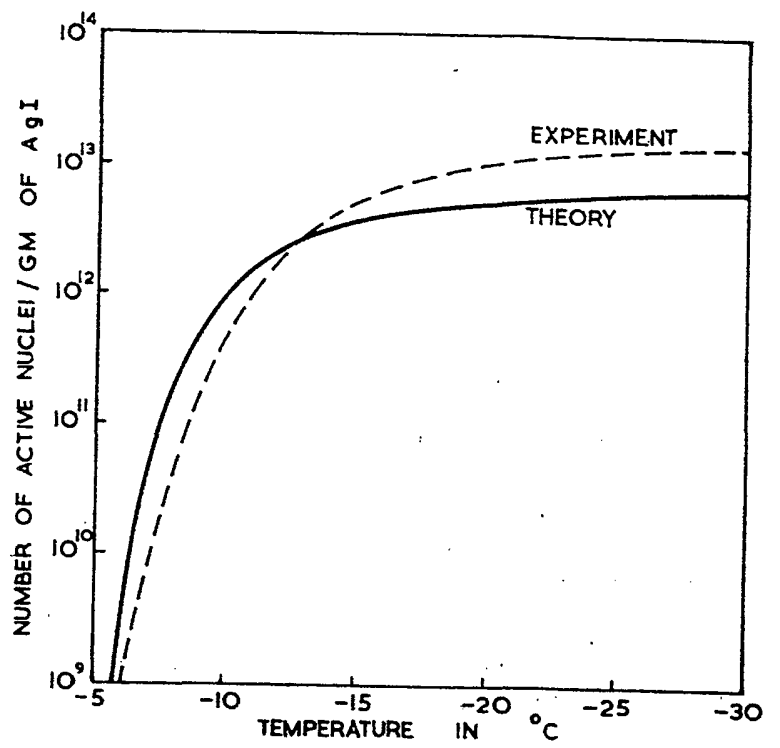

FIG. 7. A typical activity curve for silver-iodide smoke (Smith and Heffernan, 1954) and the activity curve calculated for an approximation to the particle-size distribution of this smoke. The theoretical curve has been adjusted vertically for approximate, fit.

size. Details will, of course, vary from one case to another.

Freezing.-A comparison of figs. 2 and 4 shows that the size effect is much less pronounced in freezing than it is in sublimation. The experimental situation is, however, complicated by the need for using small droplets and by the solubility of the suspended particles.

Bayardelle (1955) has filtered suspensions of silver iodide in water and determined their freezing points. The pore sizes are mostly quite large and variations in freezing point are exhibited even for filters passing all particles less than $30 \mu$. The results of this experiment are not in good agreement with our theory, but as noted above. the experimental situation is complicated.

Other experiments simply determine freezing-point ranges and give no information on which we can usefully comment here.

\section{Natural nuclei}

An understanding of the behavior of naturally occurring nuclei in the air is of considerable importance. Counts of the number of nuclei active at various temperatures have been carried out in this laboratory for several years and indicate an overall approximately exponential increase in the number of active nuclei as the temperature is decreased. The rate of increase is a factor of 10 for between $3 \mathrm{C}$ and $6 \mathrm{C}$ temperature drop.

This may be attributed in part to a size effect, but it seems likely that much of the increase may be due 
to the relative abundance of nuclei of low efficiency. This tendency is indicated by the measurements of Schaefer (1949) on the activity of various dust; ash, and soil particles.

Atmospheric deactivation processes which may be expected to have more pronounced effects on efficient nuclei than on particles with little activity are also likely to contribute to the establishment of an approximately exponential distribution as observed.

\section{Nucleation time lag}

Vonnegut (1949) observed that when silver-iodide smoke is injected into a supercooled cloud some ice crystals form almost immediately, but others continue to form at an ever decreasing rate for periods up to an hour. The decrease in rate was found to follow an exponential law with a time constant of approximately five minutes.

Warner and Newnham (1958) have observed a similar effect with natural nuclei, observing in addition that 20 to 50 per cent of the ice crystals are produced within the first ten seconds and that the same situation exists over a considerable temperature range. They found an approximately exponential behavior with a time constant of about six minutes.

Reiss (1950) has attributed Vonnegut's results to a size effect without explaining them in detail. In another publication (Fletcher, 1958b), the present author has discussed the atmospheric case in detail and shown that the observed facts can be completely explained in terms of the nucleation theory of section 2 and the observed activity distribution of the atmospheric nuclei. An almost exactly similar discussion applies to silver-iodide nuclei and accounts for the agreement between the two apparent.time constants.

Suppose we have $N_{0}$ nuclei whose nucleation rate is given by $J \sec ^{-1}$ (see equations 11 and 12), then the number of ice crystals formed after $t$ seconds is

$$
N=N_{0}[1-\exp (-J t)]
$$

These $N_{0}$ nuclei are identical and are, in fact, characterized by a temperature $T_{0}$ at which their nucleation rate $J$ is $1 \mathrm{sec}^{-1}$. At any temperature $T$, the nucleation rate of these particles will be denoted by $J\left(T_{0}, T\right)$.

Suppose, now, that instead of just one species of nuclei with characteristic temperature $T_{0}$ we have a whole distribution with different characteristic temperatures. This population can be defined by a density function $N_{0}\left(T_{0}\right)$ and, at a given temperature $T$, the number of ice crystals nucleated after time $t$ is given by the integrated form of (16).

$$
N=\int_{0}^{273} N_{0}\left(T_{0}\right)\left\{1-\exp \left[-J\left(T_{0}, T\right) t\right]\right\} d T_{0} .
$$

This integration has been performed numerically (Fletcher, 1958b) for $N_{0}\left(T_{0}\right)$, a simple exponential population of nuclei of equal size. It is found that for typical atmospheric populations a certain number of ice crystals appear within a few seconds, and the number produced increases by a factor from 2 to 5 when counting is continued for $20 \mathrm{~min}$. The count goes on increasing after this but at such a slow rate as to be negligible. If the increase is fitted to a decreasing exponential, then the time constant is about six minutes. This is not, however, an accurate description of the count increase and has no special physical significance.

The treatment can be applied with little change to the case of AgI smoke. The slight modifications due to the size of the nuclei have little effect, and the saturation of the activity near $-20 \mathrm{C}$ will improve the exponential approximation.

It is to be expected that an effect of this sort will occur in all nucleation processes involving a nonhomogeneous aerosol as the nucleating agent.

\section{Conclusion}

We have seen how an application of classical nucleation theory can help in the explanation of many aspects of the behavior of aerosol particles as icecrystal nuclei. It enables us to distinguish between action as sublimation and as freezing nuclei and to predict which process will occur under given conditions. The size effect can be calculated to a good approximation, and it also explains the observed distribution of activity in smokes. This, in turn, givés an adequate explanation of the time-lag phenomenon which has been observed in the nucleation process.

It must be emphasized, however, that this treatment merely provides a classical background for the study of these phenomena. The nucleation process itself requires further study in connection with surface free energies, adsorption, and the probable roles of surface imperfections. Similarly, the effects of surface and volume contamination are largely uninvestigated and are certain to be of great practical importance in cloud-physics studies.

\section{REFERENCES}

Bayardelle, M., 1955 : Influences des dimensions des noyaux de congélation sur la temperature de congélation de l'eau. Acad. Sci. Paris, Compt. Rend., 241, 232-233.

Birstein, S. J., 1954: The role of adsorption in heterogeneous nucleation, I : Adsorption of water vapour on silver iodide and lead iodide. J. Meteor., 12, 324-331.

Dunning, W. J., 1955 : Chemistry of the solid state. London, Butterworths, 159-183.

Fletcher; N. H., 1958a: Size effect in heterogeneous nucleation. J. chem. Phys., 29, 572-576.

Fletcher, N. H., 1958b: Time lag in ice crystal nucleation in the atmosphere. Part II-Theoretical. Bull. Obs. Puy de Dôme, 11-18. 
Glasstone, S., 1947: Thermodynamics for chemists. New York, van Nostrand Co. Inc., p. 205.

Howell, W. E., 1949: The growth of cloud droplets in uniformly cooled air. J. Meteor., 6, 134-149.

Krastanow, L., 1940 : Über die Bildung der unterkühlten Wassertropfen und der Eiskristalle in der freien Atmosphäre. Z. Meteor., 57, 357-371.

Reiss, H., 1950: Time lag in the nucleation of supercooled water clouds by silver iodide smokes. J. chem. Phys., 18, 529-533.

Sano, I., Y. Fujitani, and Y. Maena, 1956: An experimental investigation on ice nucleating properties of some chemical substances. J. meteor. Soc. Japan, Series. II, 34, 104-110.

Sano, I., and N. Fukuta, 1956: Effects of water vapour, ammonia and hydrogen sulphide against the decay of silver iodide smoke under irradiation of ultraviolet light. J. meteor. Soc. Japan, Series, II, 34, 34-40.

Schaefer, V. J., 1949: The formation of ice crystals in the laboratory and the atmosphere. Chem. Rev., 44, 291-320.

Smith, E. J., and K. J. Heffernan, 1954: Airborne measurements of the concentration of natural and artificial freezing nuclei. Quart. J. r. meteor. Soc., 80, 182-197.

Soulage, G., 1955: Etude de générateurs de fumées d'iodure d'argent. Bull. Obs. Puy de Dôme, 1-8.

Turnbull, D., and Vonnegut, B., 1952: Nucleation catalysis. Ind. and eng. Chem., 44, 1292-1298.

Vonnegut, B., 1949: Nucleation of supercooled water clouds by silver iodide smokes. Chem. Rev., 44, 277-289.

Warner, J., and T. Newnham, 1958: Time lag in ice crystal nucleation in the atmosphere. Part I-Experimental. Bull. Obs. Puy de Dôme, 1-10. 